\title{
Network meta-analysis of the effectiveness of various drugs in preventing allergic hypersensitivity in patients after anesthesia.
}

\author{
Danfeng Yuan', Pingang Li $^{2}$, Ao Yang1, Wei Dai ${ }^{1}$, Jihong Zhou ${ }^{\text {* }}$ \\ ${ }^{1}$ State Key Laboratory of Trauma, Burns and Combined Injury, Institute of Surgery Research, Daping Hospital, Army \\ Medical University, Chongqing, China \\ ${ }^{2}$ Department of General Surgery and Center of Minimal Invasive Gastrointestinal Surgery, Southwest Hospital, Army \\ Medical University, Chongqing, China
}

\begin{abstract}
Objective: To explore the effect of different drugs on postoperative hyperalgesia in general anesthesia patients.

Methods: A randomized controlled trial was conducted to detect the hyperalgesia of patients with general anesthesia from PubMed, Embase, Medline, CNKI and Wanfang databases. The visual analogue scale (VAS) assessment of hyperalgesia patients after anesthesia was extracted as well as the effects of various prophylactic agents on the incidence of side effects in postoperative patients. Multivariables meta-analysis was performed by Stata 14.0 software to explore the effectiveness of various drugs in preventing postoperative hyperalgesia and side effects.

Results: A total of 12 articles included 983 patients were included in this study. The results showed that the selected drugs had a certain effect on the prevention of hyperalgesia after anesthesia, but the incidence of side effects caused by dexmedetomidine and diazepam was the lowest.

Conclusion: From the prevention of hypersensitivity after anesthesia and reduce the incidence of postoperative side effects, it is recommended that the clinical preferred dextromethorphan, followed by the selective application of diazepam or flurbiprofen ester.
\end{abstract}

Keywords: Postoperative anesthesia, Hyperalgesia, Network meta-analysis.

Accepted November 27, 2017

\section{Introduction}

Opioids have a good analgesic effect for moderate to severe pain. Remifentanil is an ultramicro-opioid receptor agonist commonly used in clinical anesthesia, having such advantages as rapid onset, no liver and kidney metabolism, and strong controllability. However, its continuous administration to patients is easy to cause hyperalgesia after anesthesia and other adverse reactions [1]. Moreover, experimental studies have reported that opioids may cause increased susceptibility to detrimental stimuli, a sensory response to harmless irritation, that is, abnormal pain; prolonged exposure to opioid effects will prolong the duration of hyperalgesia [1-3]. A similar phenomenon also exists in opioid tolerant patients. For example, if remifentanil is administered at a constant rate for volunteers with analgesic tolerance, the duration of pain allergy is prolonged by about one hour [4]. Therefore, how to effectively prevent hyperalgesia experience in patients after general anesthesia has an important value to be explored for the clinical choice of appropriate preventive drugs.

Flurbiprofen is a non-steroidal antiinflammatory drug carried by lipid microspheres that can be directionally pooled at the incision and inflammation site to achieve the effect of targeted inhibition of pain [5]. Dexmedetomidine is an effective $\alpha 2$ adrenergic receptor agonist with sedative, analgesic and anxiolytic effects that are suitable for sedation during intensive care or intubation before anesthesia. In addition, there is
Ketamine, Tramal, Dezocine, Fentanyl, etc. However, these narcotic drugs to prevent hyperalgesia still have certain inconsistency, so that clinicians make choices with large subjective factors, which is not conducive to effective prevention of hyperalgesia after anesthesia. Therefore, the main purpose of this study is to explore the effectiveness of multiple drugs by multivariate meta-analysis.

\section{Research methods}

\section{Literature search}

The two authors searched PubMed, Embase, Medline, CNKI and Wanfang database. The search time was limited to January 2005 - January 2017. English search terms include "hyperalgesia" "Fentanyl", "Dezocine", "Tramal", "Dexmedetomidine", "Parecoxib", "Flurbiprofen", "postoperative", and "anaesthesia". By reading the titles and the abstracts of the literatures, they screened the literatures whose full texts should be obtained, and widely included the literatures that meet the standards by searching the similar literatures.

\section{Literature inclusion criteria}

All the included studies were required to meet the following criteria: (1) patients were anesthetized with remifentanil-based compound anesthesia; (2) the grouping methods in the studies 
Citation: Yuan D, Li P, Yang A, et al. Network meta-analysis of the effectiveness of various drugs in preventing allergic hypersensitivity in patients after anesthesia. Allied J Med Res. 2017;1(1):23-7.

were randomized and controlled; (3) all patients included had no hepatic and renal insufficiency, cardiovascular disease, alcoholism or drug addiction history and serious psychiatric history; (4) all patients included in the trial were enrolled in the context of informed consent of the Hospital Ethics Committee and the family members of the patient; (5) the subjects included in the trial were evaluated to type I-II surgery by the American Society of Anesthesiaologists (ASA).

\section{Information extraction}

The authors were asked to extract the information needed by this study, including the first author of the literature, the year of publication, the side effects after anesthesia, the sample size and type of surgery involved in the trial, the drug and dose used in prevention of hyperalgesia after anesthesia, the extubation time after anesthesia and the visual analogue scale (VAS) in different time periods. The extraction results that had differences were discussed between the two authors or solved by the third author.

\section{Statistical method}

The data analysis of this study was carried out in Stata 14.0 software. According to the principle of the occurrence of the probability event in frequency, the extracted data was visualized by network meta-analysis [6], and the efficacy of "fentanyl", "ketamine", "tramadol", "dexmedetomidine" and "dezocine" on hyperalgesia in the patients after anesthesia was evaluated, and all the drugs included in the analysis were ranked. The robustness of the analysis results was verified by Bayesian model.

\section{Results}

\section{Literature search results}

According to the keywords and search strategies, this study initially retrieved 537 related literatures (Figure 1). After duplicate literatures were excluded from the search results through the software NoteExpress 3.1, and after meetings, invention patents, reviews and non-randomized controls and animal tests, etc. were excluded by reading the titles and abstracts, there were a total of 14 literatures whose full texts need to be obtained. Then the literatures whose full texts cannot be obtained and that did not provide the necessary data for this study were further removed. There were 12 literatures [7-18] finally included for analysis, of which 10 was the Chinese literatures [7-12,14-17], and 2 were the English literatures $[13,18]$.

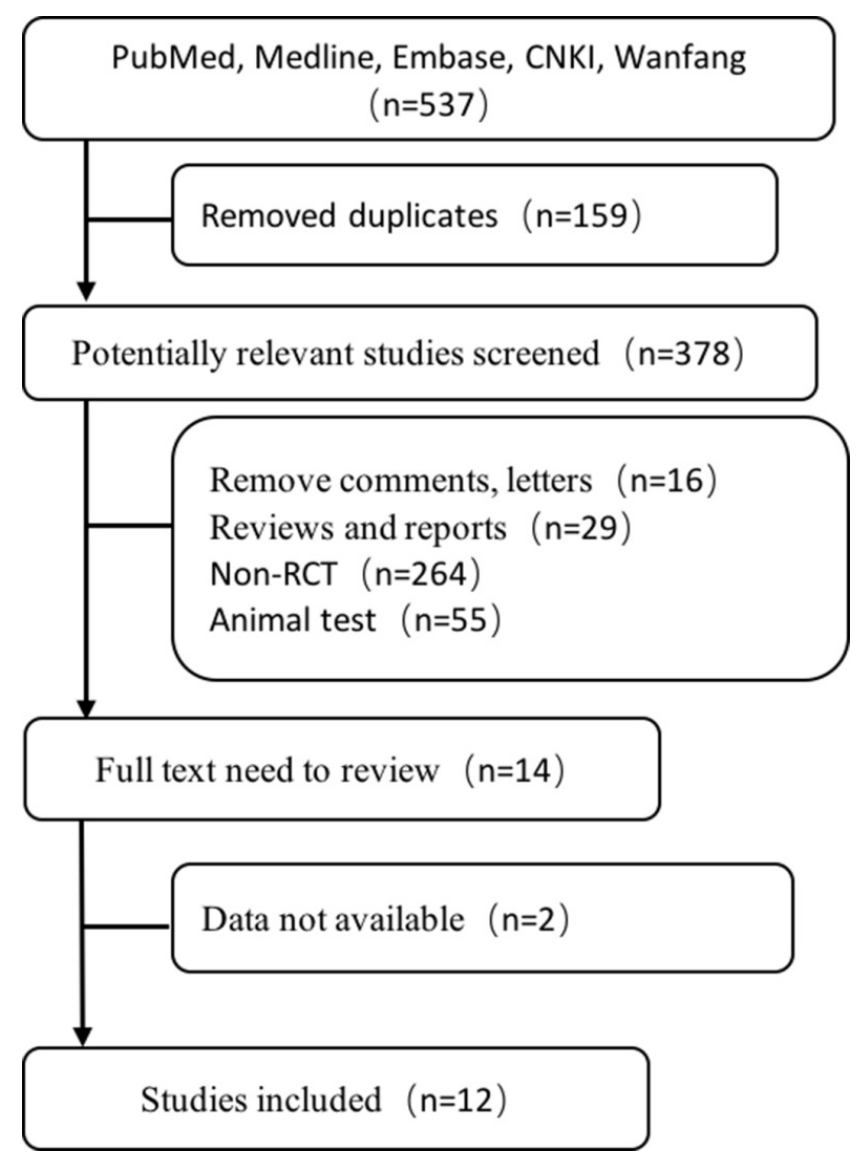

Figure 1. Flow Diagram of literature search and selection.

This study included a total of 983 cases of general anesthesia patients (Table 1), aged from 18 to 72 years. Most of the surgical types were gynecological and general laparoscopic surgery. There was no publication bias between the included literatures by the Begg method rank correlation test $(z=-0.33$, $\mathrm{P}=0.742)$ and Egger test $(\mathrm{z}=-0.43, \mathrm{P}=0.676)$.

Table 1. Characteristics of included studies.

\begin{tabular}{|c|c|c|c|c|c|c|c|}
\hline \multirow{2}{*}{$\begin{array}{r}\text { Author } \\
\text { Zhang [7] }\end{array}$} & \multirow{2}{*}{$\begin{array}{r}\text { Year } \\
2016\end{array}$} & \multirow{2}{*}{$\begin{array}{l}\text { Samples/n } \\
100\end{array}$} & \multirow{2}{*}{$\begin{array}{r}\text { Age } \\
20 \sim 60\end{array}$} & \multicolumn{2}{|c|}{ Surgical type } & \multicolumn{2}{|c|}{ Anesthetic and dosage } \\
\hline & & & & Gynecological & laparoscopic & Flurbiprofen & $2 \mathrm{mg} / \mathrm{kg}$ \\
\hline & & & & & & Dezocine & $0.2 \mathrm{mg} / \mathrm{kg}$ \\
\hline & & & & & & Flurbiprofen & $1 \mathrm{mg} / \mathrm{kg}+0.1 \mathrm{mg} / \mathrm{kg}$ \\
\hline & & & & & & Normal Saline & \\
\hline \multirow[t]{2}{*}{ Zhu [8] } & 2016 & 96 & $18 \sim 65$ & Abdominal surgery & & Flurbiprofen & $1.5 \mathrm{mg} / \mathrm{kg}$ \\
\hline & & & & & & Normal Saline & \\
\hline Li [9] & 2014 & 92 & $20 \sim 50$ & $\begin{array}{l}\text { Gynecological } \\
\text { surgery }\end{array}$ & laparoscopic & Dexmedetomidine & $1 \mu \mathrm{g} / \mathrm{kg}$ \\
\hline
\end{tabular}




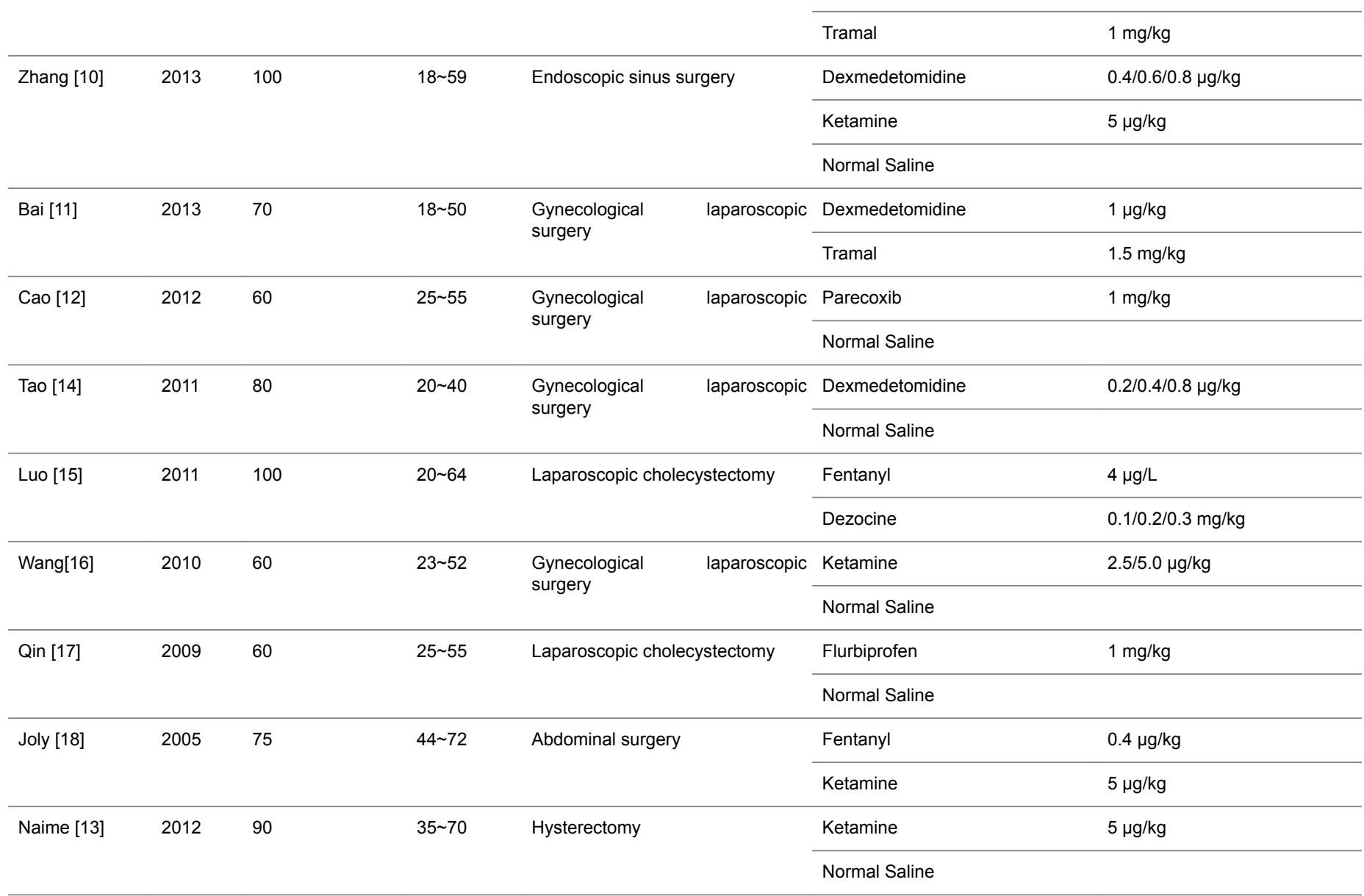
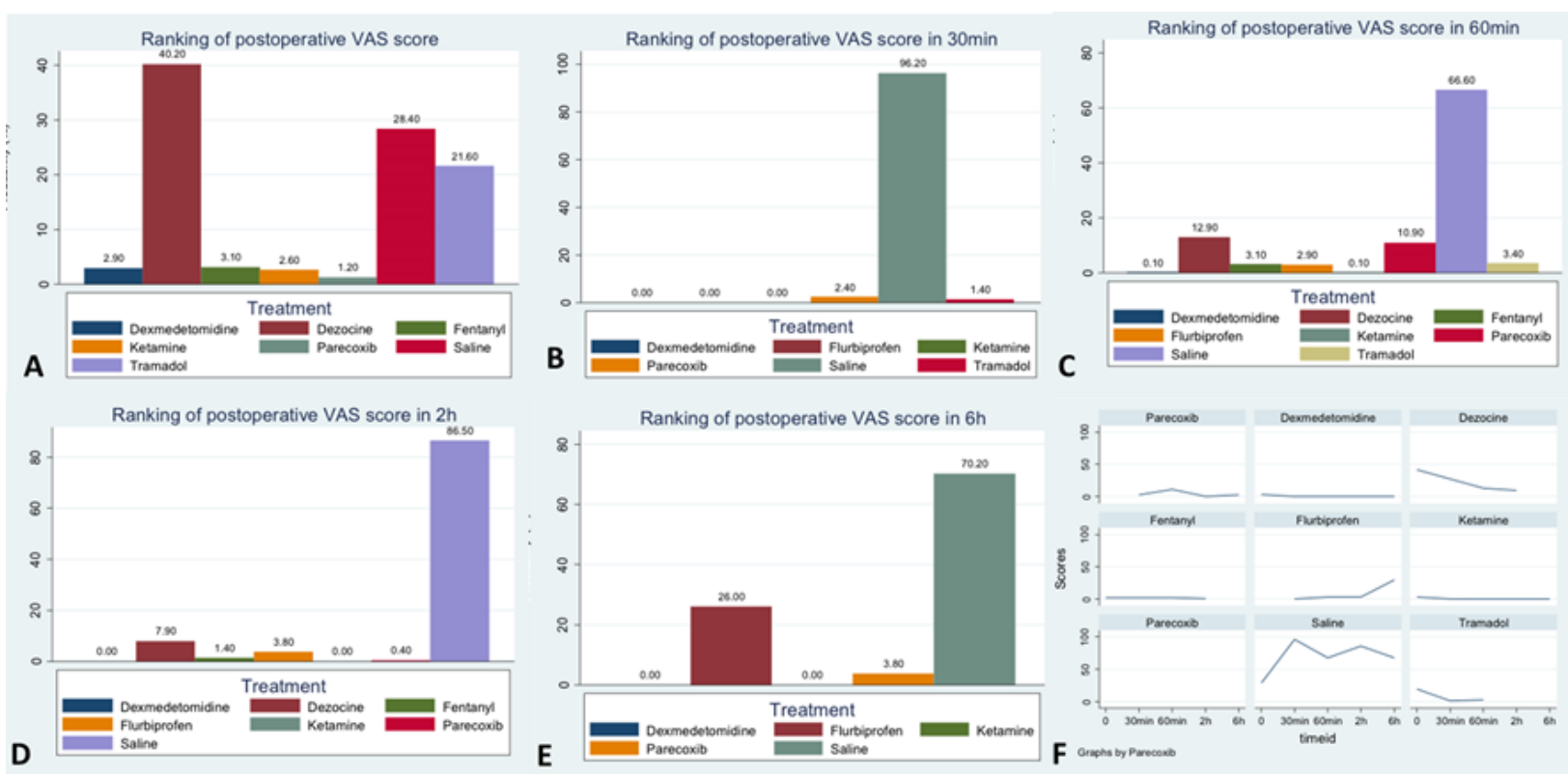

Figure 2. The ranking probabilities of VAS evaluation of different time; (A) Rank of VAS Score after treatment; (B) Rank of VAS Score 30min after treatment; (C) Rank of VAS Score 60min after treatment; (D) Rank of VAS Score 2h after treatment; (E) Rank of VAS Score 6h after treatment; (F) Rank of VAS score of different drugs at different times.

\section{Network meta-analysis results}

In this study, we analyzed the effects of various preventive hyperalgesia drugs on VAS scores according to "postoperative", "postoperative $30 \mathrm{~min} "$, "postoperative 60 min", "postoperative $2 \mathrm{~h}$ ", and "postoperative $6 \mathrm{~h}$ ", due to the differences in postoperative time points reported by each study (Figure 2A), respectively. Meanwhile, we analyzed their effects on postoperative extubation time and side effects, and 
Citation: Yuan D, Li P, Yang A, et al. Network meta-analysis of the effectiveness of various drugs in preventing allergic hypersensitivity in patients after anesthesia. Allied J Med Res. 2017;1(1):23-7.

compared the advantages and disadvantages of the selected drugs.

We found that almost all of the selected drugs had a minimum VAS score at $30 \mathrm{~min}$ through network meta-analysis on multiple preventive drugs at different time points. Dextromethorphan and ketamine showed a better preventive effect as a whole (Figures 2A-2E), and their postoperative scores at different time points were stable, basically maintained at 0 level (Figure 2F). They were followed by parecoxib and fentanyl. Tramadol and digoxin had poor starting effect, whereas flurbiprofen was effective at the outset, but with a shorter maintenance time. In addition, during the analysis of the postoperative extubation time and the incidence of side effects, we found that the mean duration of postoperative extubation of parecoxib and flurbiprofen was the shortest (Figure 3A) except for the physiological saline in the control group. The incidence of side effects of dexmedetomidine and dezocine was low (Figure 3B).
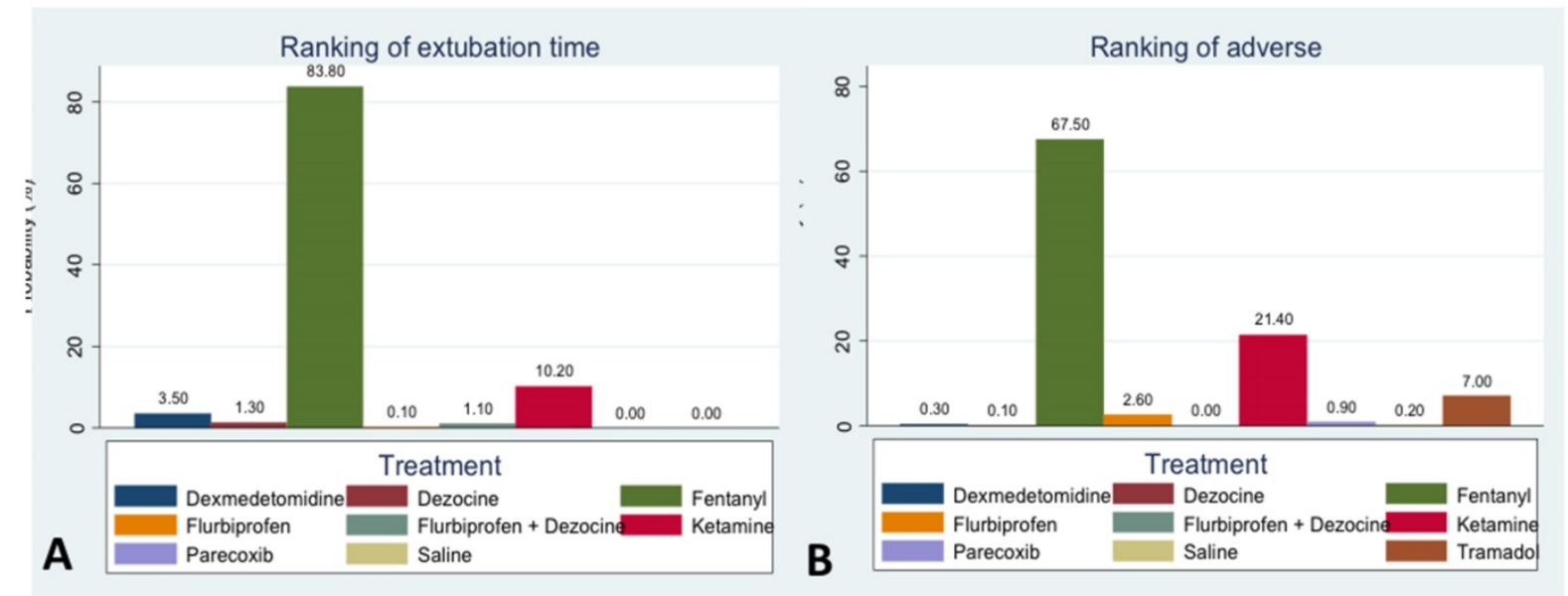

Figure 3. The ranking probabilities of extubation time and side effects of postoperative anesthesia.
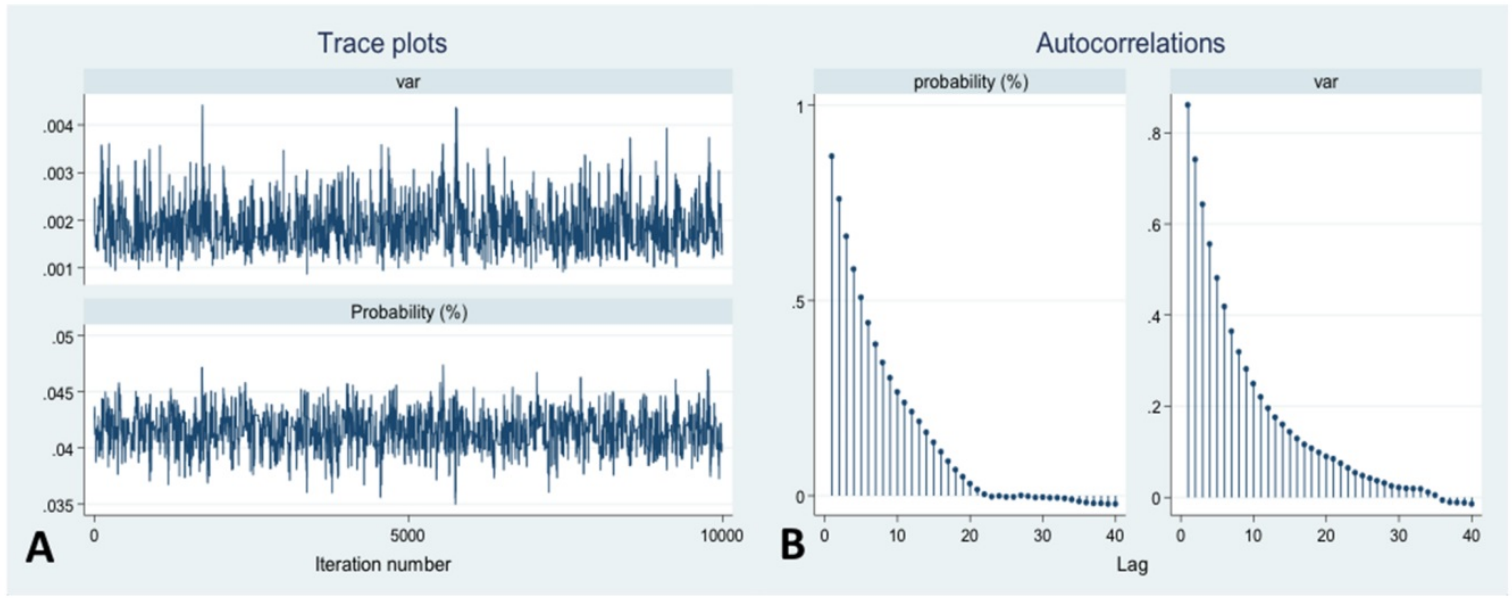

Figure 4. The Bayesian models to examine the distribution of probability and variance.

Finally, we have performed a Bayesian model test on the results of this study (Figure 4). It is shown that the convergence of the probability distribution and variance variation is better (Figure 4A) and the autocorrelation is also higher (Figure 4B) [19]. Thus, the results of this study reflect, to a certain extent, the practical efficacy of the included drugs in the prevention of hyperalgesia after anesthesia.

\section{Conclusion and Discussion}

According to the results of our multivariate network metaanalysis, we can draw the following conclusions: (1) the selected hyperalgesia prevention drugs can reduce the VAS score at different postoperative periods to varying degrees; (2)
Judging from the comprehensive results of VAS scores at different postoperative time points, the extubation time and the incidence of side effects, dexmedetomidine in the prevention of hyperalgesia after anesthesia is the best; (3) the patients who cannot tolerate postoperative side effects (Such as nausea and vomiting, drowsiness, dizziness, etc.) can be given priority to the use of dextromethorphan or dezocine, followed by the selective application of flurbiprofen or paracetin.

Previous studies have shown that there is a significant hyperalgesia area around the surgical incision [20], but the current study is to assess the extent to which opioids can affect the generation of hyperalgesia. Animal experiments show that, after the use of high doses of remifentanil, the anesthetic potency of morphine significantly drops in reducing the 
hyperalgesia around the surgical incision, which is important for the prevention of postoperative hyperalgesia [21]. In addition, studies have shown that high doses of remifentanil can increase pain sensitivity after anesthesia [18]. However, studies have shown that there is no significant difference in the demand for analgesic drugs after the application of low-dose and high-dose remifentanil [22]. Therefore, this study did not discuss the dosage of remifentanil, which is one of the study directions to be explored in the next step of the research team.

For the first time in this study, multivariate network metaanalysis was used to analyze the advantages and disadvantages of various anesthetic drugs in preventing hyperalgesia after anesthesia, which has a good guiding role in many selective drugs. In addition, there are still limitations in this study, because such confounding factors as differences in surgical types, differences in gender, and differences in the concentrations of prophylactic agents used are involved. Therefore, to strengthen the conclusions of this study, there is still a need to increase the rigor of the inclusion criteria and to expand the overall sample size.

\section{References}

1. Ruggiero G, Di Gennaro D, Vivona L, et al. Intraoperative remifentanil might influence pain levels in the immediate post-operative period after major abdominal surgery: 14AP1-10. Eur J Anaesth. 2007;24:171.

2. Koppert PW. Opioidvermittelte Analgesie und Hyperalgesie. Der Schmerz. 2005;19:386-94.

3. Vanderah TW, Ossipov MH, Lai J, et al. Mechanisms of opioid-induced pain and antinociceptive tolerance: descending facilitation and spinal dynorphin. Pain. 2001;92:5-9.

4. Duan C, Li T, Liu L. Efficacy of limited fluid resuscitation in patients with hemorrhagic shock: a meta-analysis. Int $\mathrm{J}$ Clin Exp Med. 2015;8:11645-56.

5. Hao J, Wang K, Shao Y, et al. Intravenous flurbiprofen axetil to relieve cancer-related multiple breakthrough pain: a clinical study. J Palliat Med. 2013;16:190-92.

6. Chaimani A, Salanti G. Visualizing assumptions and results in network meta-analysis: The network graphs package. Stata J. 2015;15:905-50.

7. Zhang Lin-lin, Zhao Qi, Wang Xin, et al. Effect of flurbiprofen axetil combined with diazoxyn on postoperative hyperalgesia in patients with remifentanil combined anesthesia. Chinese J Anesth. 2016;36:392-5.

8. Xiao-chang Z, Lian-jun F, Shuang W, et al. Prevention of hyperalgesia after remifentanil anesthesia with flurbiprofen axetil. J Clin Anesth. 2016;32:889-91.

9. Qing-zhong L, Shu-ling Y, Xi-e W. Clinical study of dexmedetomidine combined with tramadol in the prevention of hyperalgesia after remifentanil anesthesia. Chinese J General Pract. 2014;29:3514-517.

10. Wei Z, Li-jun Z, Quan-cheng K, et al. Comparison of the effects of different doses of dexmedetomidine and low- dose ketamine on hyperalgesia after remifentanil combined anesthesia. J Clin Anesth. 2013;29:435-8.

11. Jie B, Xiaojuan W, Hongfei X, et al. Clinical study of dexmedetomidine combined with tramadol in the prevention of hyperalgesia after remifentanil anesthesia. Northwest Pharmacy. 2013; 6:3514-17.

12. Yan C, Yu-sheng L, Xiao-feng S. Preparation of remifentanil after anesthesia in patients with gynecological laparoscopic surgery. J Clin Anesth. 2012;28:564-6.

13. Yalcin NSTUR. A Comparison of Ketamine and Paracetamol for Preventing Remifentanil Induced Hyperalgesia in Patients Undergoing Total Abdominal Hysterectomy. Int J Med Sci. 2012;9:327.

14. Jia T, Xiao-ping G, Liang-yu $P$, et al. Effects of premedication of dexmedetomidine on postoperative pain and hyperhidrosis of remifentanil. J Clin Anesth. 2011;27:947-9.

15. Hong L, Heng Y, You-yang H, et al. Effect of digoxin on postoperative hyperalgesia in patients with remifentanil combined anesthesia. Chinese J Anesth. 2011;31:1213-6.

16. Ke-man W, Xin M, Yi-feng L, et al. Effects of different doses of ketamine on hyperalgesia after remifentanil anesthesia. Hebei Medicine. 2010;32:424-5.

17. Zhong Q, Min Y, Qing A. Preparation of flurbiprofen fat in the prevention of hyperalgesia after remifentanil anesthesia. J Pract Med. 2009;25:1887-8.

18. Mei ZB, Duan CY, Li CB, et al. Prognostic role of tumor PIK3CA mutation in colorectal cancer: a systematic review and meta-analysis. Ann Oncol. 2016;27:1836-48.

19. Brannick MT. Implications of empirical Bayes metaanalysis for test validation. J Appl Psychol. 2001;86:468-80.

20. De KM, Lavand'Homme P, Waterloos H. 'Balanced analgesia' in the perioperative period: is there a place for ketamine? Pain. 2001;92:373-80.

21. Laulin JP, Maurette P, Corcuff JB, et al. The role of ketamine in preventing fentanyl-induced hyperalgesia and subsequent acute morphine tolerance. Anesth Analg. 2002;94:1263.

22. Lenz H, Raeder J, Hoymork SC. Administration of fentanyl before remifentanil-based anaesthesia has no influence on post-operative pain or analgesic consumption. Acta Anaesth Scand. 2008;52:149-54.

\section{*Correspondence to:}

Jihong Zhou,

Daping Hospital, State Key Laboratory of Trauma,

Institute of Surgery Research, Army Medical University,

Yuzhong District, Chongqing, China

Tel: 13983827031

E-mail: zhoujihong0213@163.com; traumazjh@126.com 